\title{
Rescue of a patient with a $50 \mathrm{~cm}$ broken PICC tube retracted and wrapped in pulmonary artery: a case report
}

Case report

\author{
Sha Yang ${ }^{a}$, Ren-Tao Yu ${ }^{b}$, Hui-Lan Zhang ${ }^{a}$, Xue Jianga , Jian-Ping You ${ }^{a, *}$ \\ ${ }^{a}$ Department of Infectious Diseases, Southwest Hospital, Third Military Medical University (Army Medical University), Chongqing 400038, China \\ ${ }^{b}$ Pulmonary Department, The General Hospital of Western Theater Command, Chengdu, Sichun 610083, China
}

Received: 23 December 2020; Accepted: 18 Februray 2021; Published: 20 December 2021

\begin{abstract}
Objective: With the increasing use of a peripherally inserted central catheter (PICC) in clinical application, the breaking of the PICC is increasing as well, which would turn into the emboli and drift to the heart and pulmonary artery, causing severe consequences. However, few cases have been reported on the rescue of patients with a broken PICC.

Patient concerns: A 33-year-old man, diagnosed with chronic hepatitis B-related decompensated cirrhosis and cryptococcal meningitis, was treated with amphotericin B combined with flucytosine and fluconazole by means of PICC catheterization. The patient was discharged with a catheter; when he returned for re-examination, a 50cm length of PICC broke and slipped into the vein after his sudden dysphoria. First aid was immediately administered, and then the intervention therapy to extricate the tube, including pulmonary artery angiography and intravascular removal of foreign matter, was performed based on the consensus of the in-hospital vein treatment group. At last, the broken PICC fragment was successfully taken out of the vessel. Re-examination after surgery showed that he recovered well.

Conclusions: Once the catheter is broken, the X-ray examination should be performed at the first instance and re-examined frequently. Moreover, the involvement of a multidisciplinary team should be formed to decide the appropriate method of treatment to ensure a successful rescue.
\end{abstract}

Keywords: case report $\bullet$ fracture $\bullet$ gooseneck catcher $\bullet$ PICC $\bullet$ pigtail catheter

(c) Shanxi Medical Periodical Press.

\section{List of Abbreviations}

PTT, activated partial thromboplastin time; ALT, alanine aminotransferase; Alb, albumin; HG, hemoglobin; INR, international normalized ratio; PICC, peripherally inserted central catheter; PLT, platelet count; PTA, prothrombin activity; PT, prothrombin time; RBC, red blood cell; TBIL, total bilirubin; WBC, white blood cell count.

\section{Introduction}

A peripherally inserted central catheter (PICC) refers to the catheter that is inserted into the deep vein from the peripheral vein (cutaneous vein, medial cubital vein, cephalic vein) and has its distal end located in the superior vena cava or subclavian vein. With the increasing use of PICC in clinical application, complications raised

How to cite this article: Yang S, Yu RT, Zhang HL, Jiang X, You JP. Rescue of a patient with a $50 \mathrm{~cm}$ broken PICC tube retracted and wrapped in pulmonary artery: a case report. Front Nurs. 2021; 4:445-450. 
by the PICC are increasing as well. It is reported that the incidence of broken PICC is $1 \%-2 \% .^{1,2}$ Once broken, the catheter would turn into emboli and drift to the heart and pulmonary artery, which causes systemic symptoms like headache, shortness of breath, unconsciousness, pale skin, tachycardia, and high blood pressure with an incidence rate of $6.7 \%$. Pulmonary embolism or other types of thromboembolism are emergent and life-threatening diseases requiring immediate rescue. Here, we report a successful rescue of a patient with a $50 \mathrm{~cm}$ length of broken PICC tube retracted and wrapped in the pulmonary artery. This study was approved by the ethics committee of Southwest Hospital, Chongqing, China. Informed consents were obtained from the parent. The study protocol conforms to the ethical guidelines of the 1975 Declaration of Helsinki.

\section{Case presentation}

This case was drafted and outlined in accordance with the care guidelines, which regulate the discipline of case reports. A 33-year-old man presented with serum HBsAg positive for 14 years, repeatedly fatigue, anorexia, jaundice for 9 years, and headache, abdominal pain for 8 days on September 26, 2017. Before hospitalization, he was treated with adefovir dipivoxil for 1 year, and then he was treated with lamivudine plus tenofovir dipivoxil for 3 years. Physical examinations revealed phase of chronic liver disease, skin and sclera jaundice, and positive abdominal mobility dullness. During blood tests, the liver function was mildly abnormal, white blood cell count (WBC) was $2.92 \times 10^{9} / \mathrm{L} \downarrow$, the hemoglobin (HCG) level was $69 \mathrm{~g} / \mathrm{L} \downarrow$, and the platelet count $(\mathrm{PLT})$ was $28 \times 10^{9} / \mathrm{L} \downarrow$. Liver ultrasound finding suggested liver cirrhosis, splenomegaly (75 mm), portal hypertension, and ascites (48 mm).

After hospitalization, the patient received lumbar puncture. The cerebrospinal fluid pressure was $350 \mathrm{~mm}$ $\mathrm{H}_{2} \mathrm{O}$, and Cryptococcus histolyticus was detected in the cerebrospinal fluid. Based on his medical history, he was finally diagnosed with chronic hepatitis B-related decompensated cirrhosis and cryptococcal meningitis. And then, a total of $125 \mathrm{~mL}$ of $20 \%$ mannitol was prescribed to reduce intracranial pressure. Recommended by the guidelines for cryptococcosis treatment, ${ }^{3}$ and Chinese expert consensus on the diagnosis and treatment of cryptococcal meningitis, ${ }^{4}$ amphotericin $B$ (the dose of $10 \mathrm{mg}$ per day was increased to $100 \mathrm{mg}$ per day) combined with flucytosine and fluconazole were selected both in the induction phase and consolidation phase. According to the guidelines, the total duration of treatment lasted for at least 10 weeks and may reach 1-2 years if necessary. Meanwhile, antiviral treatment with lamivudine plus tenofovir dipivoxil was administered at the same time. Considering the long-term intravenous administration of drugs, PICC catheterization was performed on the right cephalic vein of the patient on September 30. On November 26, for personal reasons, the patient returned to his local hospital along with the catheter in place after the precautions of PICC-related nursing care were explained to him.

On December 1st, the patient's condition worsened with severe headache, high fever, and vomiting. The cerebrospinal fluid pressure by lumbar puncture was $400 \mathrm{~mm} \mathrm{H} \mathrm{H}_{2} \mathrm{O}$, so he was sent back to our department again. Body examination and routine nursing suggested that the PICC was fixed in the right cephalic vein safe and sound, so the treatment continued. At 19:10 on December 2, the patient's condition was further aggravated with a sudden loss of consciousness after sudden extremity spasm. Shortly afterward, his breath and heartbeat stopped and carotid pulsation disappeared. At 19:13, the patient's breath and heartbeat recovered after closed cardiac massage. But immediately, dysphoria irritability appeared, followed by yelling and crying. After the protective constraint was imposed, he left the hospital under the command of his family. On December 18th, he returned for re-examination, but only the fixed joint was found and the PICC was missing. The chest X-ray examination was then immediately performed and the image is shown in Figure 1. Based on the clinical manifestations and image examinations, it was considered that the PICC had slipped into the vein. However, the patient's family refused further treatment or rescue due to personal issues and asked strongly for leaving the hospital immediately. Having been told the possible adverse consequences of catheter detachment, the family members still strongly insisted on leaving the hospital and left the hospital after signing the consent form for giving up treatment.

On December 18, when the patient's condition was becoming increasingly stable, he repeatedly requested re-hospitalization for further treatment. It turned out that only the fixed joint was left at the PICC puncture point of the right arm but no catheter was seen. Based on this situation, we considered that the catheter was broken and left in the body, so the nurse immediately took him off the left lateral position and grasped the broken catheter outside of the body (Figure 1A) and wrapped it with tourniquet ligation (Figure 1B). During bandaging, the start time was marked, and the patient's limb peripheral circulation was closely evaluated and recorded. The chest X-ray and CT scan suggested that there was a high-density shape around the pulmonary artery, as shown in (Figure 2). The linear shadow was considered as the PICC tube.

The in-hospital vein treatment group of the Southwest Hospital was established to settle emergent or complicated situations associated with blood vessels, 


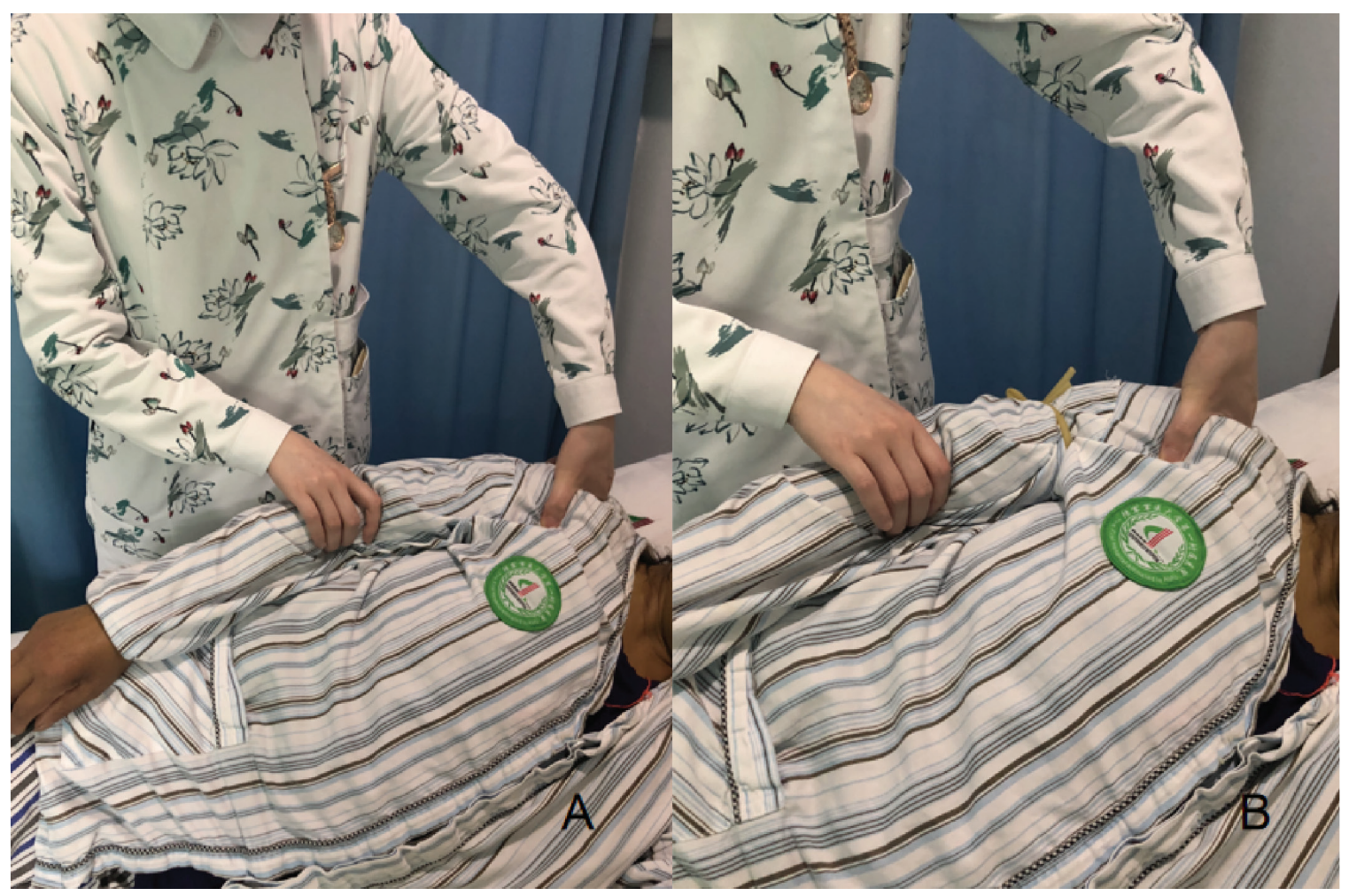

Figure 1. The method of first aid immediately after diagnostic imaging revealed that the catheter had broken off inside the body: (A) left recumbent position, hand; and (B) tourniquet ligation.

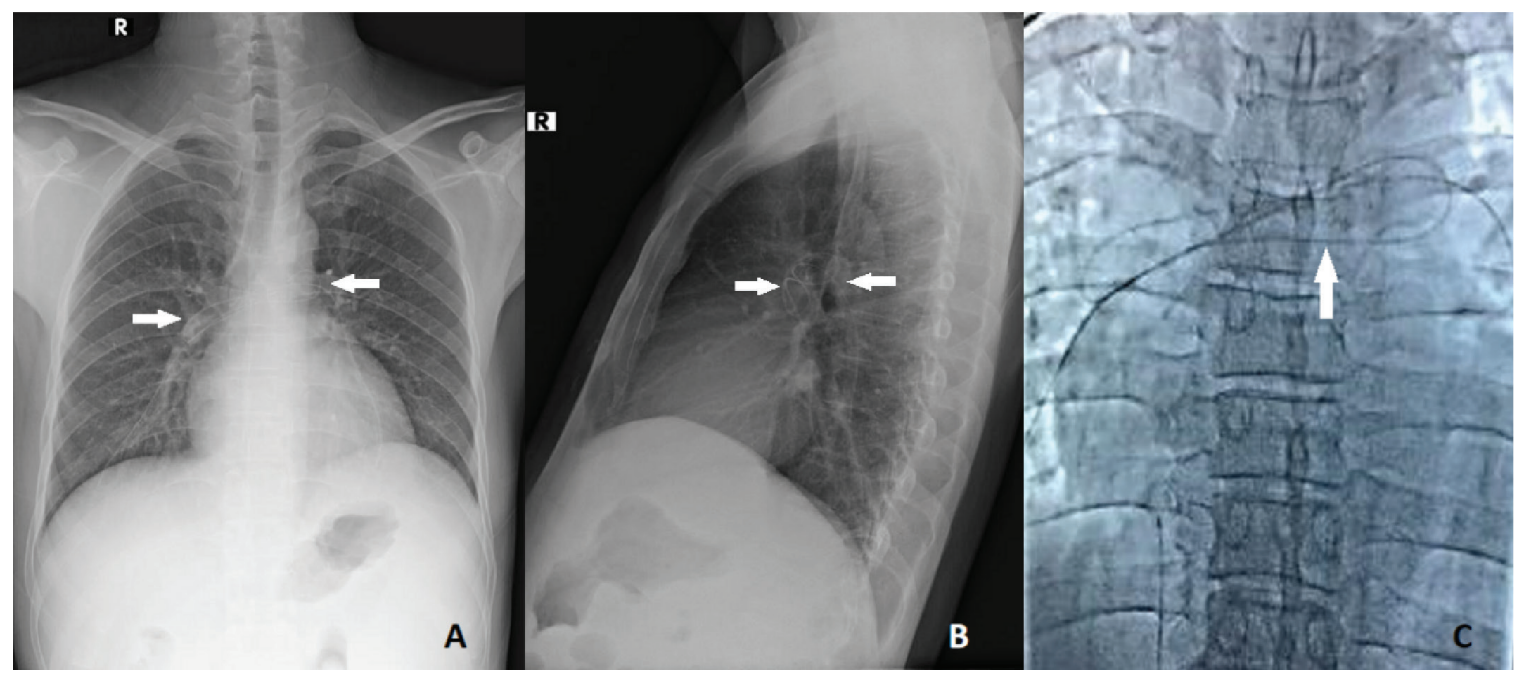

Figure 2. $X$-ray and CT scan results of the broken catheter inside the body by (A) chest positive tablets; (B) chest lateral tablets; and (C) enhanced $\mathrm{CT}$, showing that the catheter was stranded in the left and right pulmonary artery trunk and branches; the ruptured catheter is indicated by an arrow.

consisting of dozens of relevant specialists. Finally, intervention therapy, including pulmonary artery angiography and intravascular removal of foreign matter, was performed to extricate the tube, based on the consensus of the group. The surgical procedure is presented in Figure 3. Specifically, the Seldinger technique was applied to place the $5 \mathrm{~F}$-catheter sheath from the right femoral vein. After the guide wire was introduced, the sheath instrument was replaced and a Y-type valve was connected by the tail end. Then, a pigtail catheter is introduced to wrap the broken PICC and pull it out of the pulmonary artery into the inferior vena cava. However, 


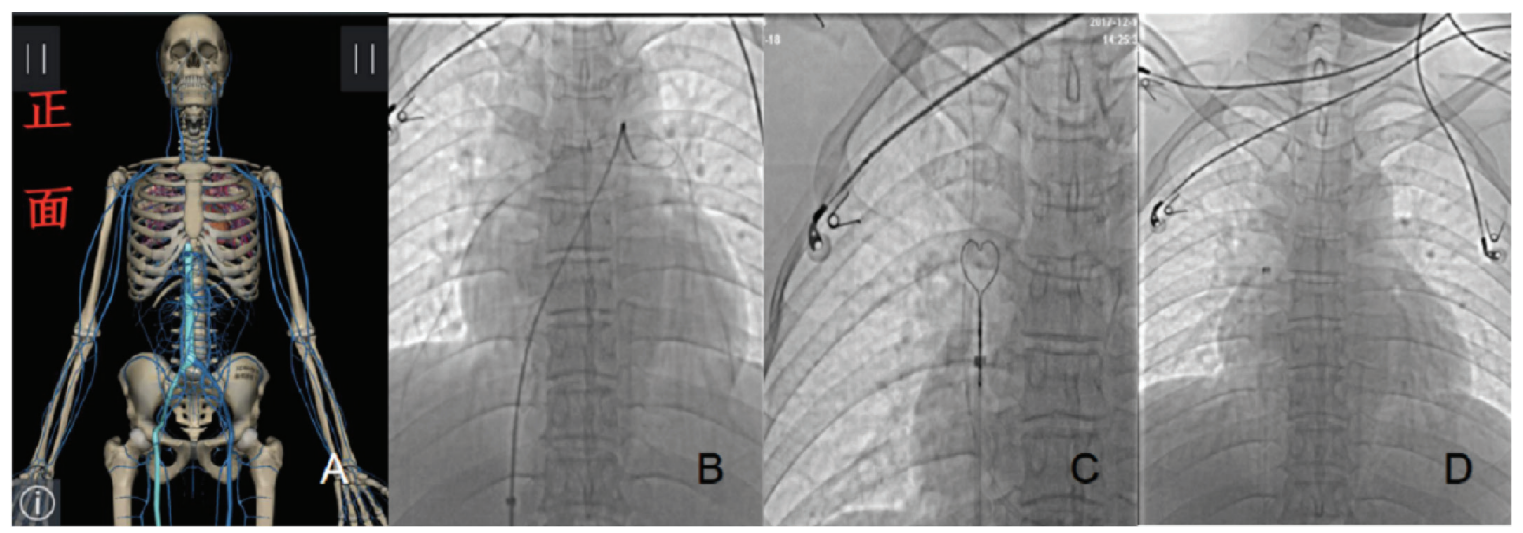

Figure 3. Images obtained during pulmonary artery angiography and intravascular removal of foreign matter by using (A) a pigtail catheter thread through the iliac vein, inferior vena cava, right atrium, and right ventricle pulmonary artery and (B) the guide wire wrapped around the fractured catheter (C) shows the extraction of the fractured catheter out of the pulmonary artery and its guidance into the inferior vena cava; and as the heart contracted, the broken tube drifted into the superior vena cava and the internal jugular vein. We inserted the goose neck snare through the iliac vein, inferior vena cava, right atrium, and superior vena cava and opened the goose neck ring waiting for the exposed free fractured catheter to drift into the ring; and (D) shows the tightening of the ring, indicating it slowly being drawn through the right atrium, inferior vena cava, iliac vein, and femoral vein. Finally, the fractured catheter is successfully removed.

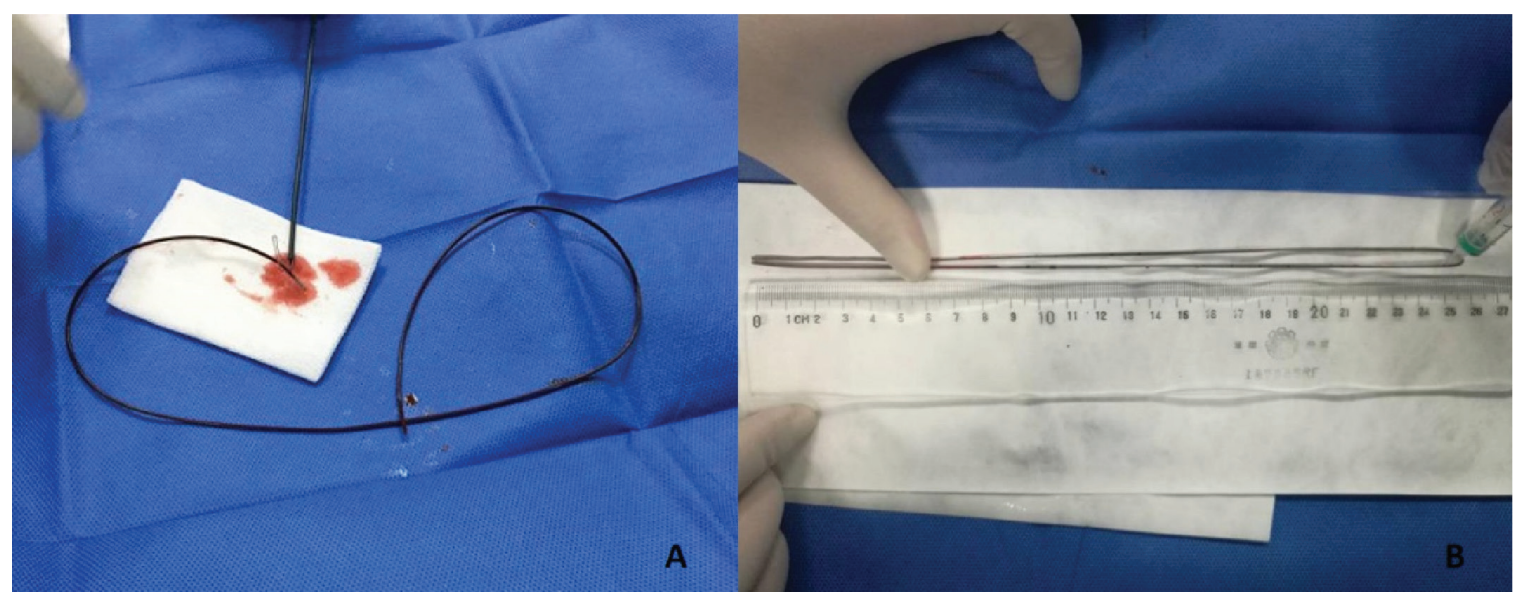

Figure 4. Images of the real broken catheter after being taken out of the body: (A) the broken catheter and (B) the length of the catheter measuring $50 \mathrm{~cm}$.

the broken PICC was found to be separated from the pigtail catheter at this place. Consequently, another intervention was given under the guide of vessel angiography. At last, the broken PICC fragment, which was $50 \mathrm{~cm}$ in length, was successfully taken out of the vessel as shown in Figure 4. After the operation, pressure dressing was performed on the right femoral vein, and the patient did not complain of any discomfort.

Two-day intravenous anticoagulation and anti-infection treatment was prescribed to the patient before postoperation re-examination. On December 20, the patient had no further discomfort, so his treatment was changed to oral medication. After operation, the patient was satisfied with the treatment procedure and discharged from our department then. Telephone follow-up after operation was performed on December 21 2017, June
12 2018, and February 11 2019, and the patient had no complain, no discomfort, and had returned to normal life and work. The specific results of blood tests at each follow-up time are listed in Table 1. We conclude that this patient had completely recovered and our rescue was successful.

\section{Discussion}

Establishing emergency plans and strengthening training are key to avoiding complications from broken tubes. Since the situation is rare when medical staff encounter that PICC is broken and remains in the vein or artery, it may result in less attention. For medical personnel, a treatment plan for the broken tube is essential to tackle this kind of emergency. Meanwhile, 
emergency drills and exercises combined with family education should be provided at the same time to avoid such emergency.

For the ultra-long catheter (catheter length $\geqq 30 \mathrm{~cm}$ ) fractured and wracked within the vessel, the correct method should be selected according to different conditions. Basically, there are two methods: venous incision ${ }^{5}$ and interventional therapy. ${ }^{6}$ Venous incision is limited to the case when the segment of the catheter is localized within the upper limb. This method causes more trauma and complications, so it has limited clinical application. An interventional method for foreign matter arrest is a more common method for taking out broken tubes from inside the body. This method is the most effective one because of less trauma and a higher success rate, was and is thus considered as the standard treatment. ${ }^{7}$ If the distal end of the catheter is located in the superior and inferior vena cava, the gooseneck ferrule could be directly introduced. Then, the end of the catheter could be pulled out from the vessel. If the catheter fragment has entered the heart chamber or the pulmonary artery, the pigtail catheter should be introduced first to hook the broken catheter end. Then, we wrap and slowly rotate the broken tube around the pigtail catheter and pull it out from the heart chamber to the inferior vena cava. After that, the gooseneck ferrule is introduced to carefully pull the broken catheter out of the body. The successful rescue of this patient validated the advantages of interventional surgery, and also, this situation was a hard test for medical personnel in making accurate decisions during emergencies.

The broken PICC remaining in the vein or artery may cause no obvious discomfort for a short period of time, but the relevant complications may occur all of sudden during the long term, which could be life threatening and emergent to the patient. The broken PICC in circulating systems may cause a series of clinical manifestations associated with catheter embolism, and some research analyzed the incidence, ${ }^{8}$ including central venous catheter insufficiency $(56.3 \%)$, arrhythmia $(13.0 \%)$, pulmonary symptoms $(4.7 \%)$, and sepsis $(1.8 \%)$, but $24.2 \%$ of patients still had no obvious symptoms. However, once catheter embolism occurs, the patient may experience severe symptoms like atrial fibrillation, dyspnea, cough, and acute chest pain. In our case, a $50 \mathrm{~cm}$ long PICC fragment drifted into the branch of the left and right pulmonary artery. But luckily enough, the patient's condition was stable enough to allow us to perform further interventional therapy successfully.

In the case of the treatment of venous catheter rupture, multidisciplinary collaboration and the leadership of the head nurse are essential. In this case, the catheter was successfully removed quickly, and the patient's 
injury was reduced to the minimum. After operation, no infection, thrombosis, or any other complications were reported during 1-year follow-up; so, it can be inferred that the patient's life was not adversely affected in the later stages following the operation. During the successful disposal process, the head nurse's ability to organize and coordinate is very important. At the same time, benefits also came from the coordination of hospital management, the standardized procedures of medical staff, and the coordination and support of the radiology department, intervention department, and hospital vein management team.

\section{Conclusions}

In conclusion, PICCs are widely used in clinical practice. Carelessness after discharge from the hospital, the lack of knowledge of catheter nursing, and negligence in relation to the broken tube after the occurrence of complications are the reasons for the retraction and entanglement in the pulmonary artery of the patient after fracture. For patients with sudden changes in their condition and consciousness, medical staff should pay special attention to the PICC fixation to avoid the occurrence of fracture. Once the catheter is broken, X-ray examination must be performed at the first time and re-examined frequently. In this case, the broken PICC was $50 \mathrm{~cm}$ in length but was successfully pulled out at last. As a result, we can conclude that broken catheter at any time should be taken out of body, on the premise that the patient did

\section{References}

1. Gnannt R, Patel P, Temple M, et al. Peripherally inserted central catheters in pediatric patients: To repair or not repair. Cardiovasc Intervent Radiol. 2017;40:845-851.

2. Jumani K, Advani S, Reich NG, Gosey L, Milstone AM. Risk factors for peripherally inserted central venous catheter complications in children. JAMA Pediatr. 2013;167:429-435.

3. Liu ZY, Wang GQ, Zhu LP, et al. Expert consensus on the diagnosis and treatment of cryptococcal meningitis. Chin J Intern Med. 2018;57:317-323.

4. Meya DB, Manabe YC, Boulware DR, et al. The immunopathogenesis of cryptococcal immune reconstitution inflammatory syndrome: not have any serious complications and stay stable. Once this occurs, the involvement of a multidisciplinary team should be organized to decide the most appropriate method of treatment to ensure a successful rescue.

\section{Acknowledgments}

We appreciate the in-hospital vein treatment group of Southwest Hospital, especially Professor Xuequan Huang and Professor Qing Mao, for technical assistance.

\section{Author contributions}

JY designed the work. $\mathrm{HZ}$ and $\mathrm{XJ}$ collected the data. SY and RY analyzed and interpreted the data. SY and RY prepared the manuscript. All authors revised the manuscript. All authors read and approved the final manuscript.

\section{Ethics approval}

Informed consent was obtained from this patient. The project was carried out in agreement with the Ethical Committee of the Southwest Hospital. Consent to publish has been obtained from the patient to report his medical information.

\section{Conflicts of interest}

All contributing authors declare no conflicts of interest.

understanding a conundrum. Curr Opin Infect Dis. 2016;29:10-22.

5. Dell'Amore A, Ammari C, Campisi A, et al. Peripheral venous catheter fracture with embolism into the pulmonary artery. J Thorac Dis. 2016;8:E1581-E1584.

6. Schechter MA, O'Brien PJ, Cox MW. Retrieval of iatrogenic intravascular foreign bodies. J Vasc Surg. 2013;57:276-281.

7. Motta LFJ, Carnevale FC, Nasser F, et al. Endovascular techniques and procedures, methods for removal of intravascular foreign bodies. Rev Bras Cir Cardiovasc. 2010;25:202-208.

8. Carroll MI, Ahanchi SS, Kim JH, et al. Endovascular foreign body retrieval. J Vasc Surg. 2013;57:459-463. 